\title{
Türkiye’de Üretilen Bazı Monofolaral Bal Örneklerinin Biyokimyasal Özelliklerinin Belirlenmesi
}

\section{Aziz Gül*}

Mustafa Kemal Üniversitesi, Ziraat Fakültesi, Zootekni Bölümü, 31034 Antakya/Hatay, Türkiye

\section{A K A L E B İ L G İ S I}

Geliş 29 Haziran 2016

Kabul 27 Ekim 2016

Çevrimiçi baskı, ISSN: 2148-127X

Anahtar Kelimeler:

Monofloral bal

Polen

Biyokimyasal analizler

Gida güvenliği

Bal standartları

*Sorumlu Yazar:

E-mail: agul25@gmail.com

\section{Ö Z E T}

Bu çalışma, Türkiye genelinde üretilen 10 farklı monofloral bal çeşidinin biyokimyasal özelliklerini belirlemek amacıyla 2013 yılında yapılmıştır. Bu çalışma için bal örnekleri arıcılardan temin edilmiş, mikroskobik incelemeler ile bal örneklerindeki dominant polenler ve dominant polenlerin baldaki temsil oranları belirlenmiştir. Polen analizleri, Hayıt (Vitex agnus-castus) ve ayçiçeği (Helianthus annuus) bal örneklerinin kendi bal örneklerini en yüksek oranda temsil ettiğini ortaya çıkarmıştır. Bu oran Hayıt (Vitex agnus-castus) ve ayçiçeği (Helianthus annuus) bal örnekleri için \%86 olarak belirlenirken en düşük polen miktarına sahip anason balı örnekleri için \%45 olarak bulunmuştur.

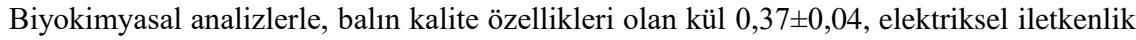

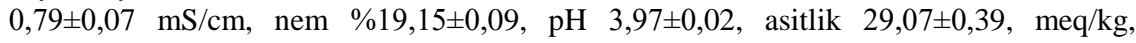

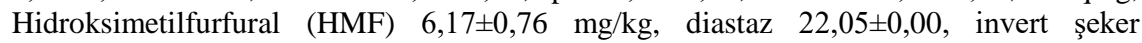
$\% 69,72 \pm 0,49$ ve sakkaroz $\% 2,90 \pm 0,30$ olarak belirlenmiştir. Çalışmanın sonunda, bal örneklerinin biyokimyasal içeriklerinin, Türk Gıda Kodeksi Bal Tebliği, Avrupa Birliği Standardı ve Kodeks Standartlarına uygun olduğu belirlenmiştir.

Turkish Journal Of Agriculture - Food Science And Technology, 4(12): 1123-1126, 2016

The Determination of the Biochemical Properties of Some Monofloral Honey Samples Produced across Turkey

\begin{tabular}{|c|c|}
\hline A R T I C LE INFO & A B S T R A C T \\
\hline $\begin{array}{l}\text { Article history: } \\
\text { Received } 29 \text { June } 2016 \\
\text { Accepted } 27 \text { October } 2016 \\
\text { Available online, ISSN: 2148-127X } \\
\text { Keywords: } \\
\text { Monofloral honey } \\
\text { Pollen } \\
\text { Chemical analysis } \\
\text { Food safety } \\
\text { Honey standards } \\
\text { *Corresponding Author: } \\
\text { E-mail: agul25@gmail.com }\end{array}$ & $\begin{array}{l}\text { This study was conducted in } 2013 \text { to determine the biochemical properties of } 10 \text { different } \\
\text { monofloral honey types produced across Turkey. For the study, honey samples were } \\
\text { obtained from beekeepers and the identity and relative percentage of dominant pollen } \\
\text { types in samples was determined through microscopic analysis. Pollen analysis revealed } \\
\text { that honey types chaste tree (Vitex agnus-castus) and sunflower (Helianthus annuus) } \\
\text { contained high proportions of each respective pollen type. These proportions reached as } \\
\text { high as } 86 \% \text {, while other honey types such as Anason had low Anasom pollen content, } \\
\text { occurring at } 45 \% \text { in whole honey samples. Biochemical analysis revealed that the average } \\
\text { honey sample quality characteristics were as followings: ash } 0.37 \pm 0.04 \text {, electricity } \\
\text { conductivity } 0.79 \pm 0.07 \mathrm{mS} / \mathrm{cm} \text {, moisture } 19.15 \pm 0.09 \% \text {, pH } 3.97 \pm 0.02 \text {, acidity } 29.07 \pm 0.39 \\
\text { meq/kg, hydroxymethylfurfural (HMF), } 6.17 \pm 0.76 \mathrm{mg} / \mathrm{kg} \text {, diastase } 22.05 \pm 0.00 \text {, invert } \\
\text { sugar } 69.72 \pm 0.49 \% \text { and sucrose } 2.90 \pm 0.30 \% \text {. These analyses confirmed that honey } \\
\text { samples met standards outlined by the Turkish Food Codex Honey Standard, European } \\
\text { Union Honey Standard and Codex Standards respectively. }\end{array}$ \\
\hline
\end{tabular}

\section{Giriş}

Bal, yüksek besin değeri ve birçok yararlı sağlık teşvik etkileri olan, dünya çapında tanınan doğal bir besindir. Balın biyokimyasal içeriği genel olarak karbonhidratlardan (kütle oranı en az \%60), tüketildiğinde hızlı bir enerji kaynağı olan glikoz ve früktoz gibi özellikle indirgeyici şekerlerden oluşur. Ayrıca bal minör bileşenler olarak aminoasitler, vitaminler, organik asitler, mineraller ve çeşitli fitokimyasallar içermektedir (Kaskoniene ve ark., 2010; Chua ve Adnan, 2014).

Balın antioksidan, antimikrobiyal, antiviral, antiparaziter, antiinflamatuar, antimutajenik ve antitümör etkileri yapılan araştırmalarda bildirilmiştir (Karadal ve Yıldırım, 2012). Bu özellikleri bala içeriğinde bulunan bazı bileşenler vermektedir. Örneğin balların antioksidan kapasitesi katalaz, fenolik asitler, proteinler, organik asitler, askorbik asit, aminoasitler, fenolik maddeler (Aljadi ve Kamaruddin, 2004; Nasuti ve ark., 2006; Vela ve ark., 2007; Wang, 2011), suda eriyen vitaminler (Chua ve Adnan, 2014) gibi bileşenlere bağlı olarak değişiklik göstermektedir. Baldaki antimikrobiyal etkinlikten ise büyük oranda hidrojen peroksit, katalaz, glikoz oksidaz seviyesinin sorumlu olduğu (Weston, 2000; White ve ark., 
1963; Silici ve ark, 2010) ya da lizozim ve fenolik maddeler gibi peroksit olmayan faktörlerin de baldaki antimikrobiyal etkinliğe katkıda bulunduğu şeklindeki bildirişler literatürde yer almaktadır (Weston, 2000; Silici ve ark., 2010).

Balın kimyasal içeriğinin korunması besin değerinin yanı sıra balın sahip olduğu terapötik özelliklerinin de korunması anlamına gelmektedir. $\mathrm{Bu}$ gün bal konusunda belirlenmiş olan ve dünya genelinde kabul gören bazı standartlara uygun bir üretim yapılmadığ 1 için balın kimyasal içeriğinin bozulması ve kalitesini kaybetmesinin yanında ekonomik anlamda da hem arıcı hem de ülke kayıplar yaşamaktadır. (Fıratlı ve Ark., 2000) Ayrıca arıcıların hastalık ve parazitlerle ve özellikle varroa mücadelesi amacıyla kullandıkları ilaçların balda kalıntı bırakması (Kaftanoğlu ve Özgür, 2000), bunların yanında, naftalin kullanımı, nişasta ihtiva eden besinlerle besleme ve balda bıraktığı kalıntılar ile C-13 testinden geçmeyen balların ihracata sunulmaması önemli sorunlar arasında yer almaktadır (Kalpaklığlu, 2000). Dünyada gerek kovan sayısı ve gerekse bal üretimi bakımından ilk 5 ülke arasında olmamıza rağmen yaşanılan bu sorunlar diş satımı olumsuz olarak etkilemiş ve dünya pazarlarında Türk ballarının imajının zedelenmesine aynı zamanda ihracattan sağlanması beklenilen önemli gelir kayıplarına neden olmuştur. Genel olarak balın \%70-80'i şekerlerden oluşmaktadır. Bu oranın \%80-90'ını glikoz ve fruktoz oluşturmaktadır (Genç ve Dodoloğlu, 2011). Başka bir deyişle balın kuru ağırlığının \%95'ini başta fruktoz ve glikoz oluşturur. Balda yaklaşık 25 farklı di-trisakkarit olmakla birlikte, total karbonhidratların toplamda \%5-10 kadarını oligosakkaritler meydana getirmektedir (Bogdanov ve ark., 2008). Baldaki şeker içeriği balın elde edildiği kaynağa ve arıların salgıladıkları enzimlerin aktivitelerine bağlıdır (Genç ve Dodoloğlu, 2011). White ve ark. (1961) yaptıkları bir çalışmada balın yaklaşık 2 yıl depolanması durumunda balda mevcut monosakkaritlerin \%9'unun kompleks disakkarit ve yüksek şekerlere dönüştüğünü belirtmişlerdir. Ayrıca bu araştırmada balların depolama süresi arttıkça yapılarındaki serbest glikoz ve fruktoz miktarı düşerken, baldaki fruktoz/glikoz oranında da artış gözlendiği rapor edilmiştir (Genç ve Dodoloğlu, 2011). Balda genel olarak fruktoz oranı glikozdan fazladır ve glikoz oda sıcaklığındaki glikoz monohidrat yapısına dönüşerek balı kristalleştirme eğilimindedir (Cavia ve ark., 2002). Fruktoz/glikoz oranı 1,0-1,2 arasında iken kristalizasyon çabuk, 1,3 veya daha fazlaysa kristalizasyon geç olmaktadır. Günümüze kadar balın biyokimyasal içeriği TSE, AB ve Kodeks gibi kuruluşlar tarafından bazı kıstaslar ile standardize edilmiştir. TSE'ye göre balın kalitesi içerdiği nem, şeker kompozisyonu, Hidroksimetil furfural (HMF), diyastaz sayısı gibi parametreler ile tayin edilmektedir.

$\mathrm{Bu}$ çalışmada Türkiye'de üretilen olan bazı monofloral balların biyokimyasal olarak incelenmesi, ulusal ve uluslararası bal standartlarına uygunluğunun belirlenmesi amaçlanmıştır.

\section{Materyal ve Metot}

$\mathrm{Bu}$ çalışmanın materyalini herhangi bir 1sıtma işlemine tabi tutulmayan 10 farklı monofloral bal örneği oluşturmaktadır. Çalışmada kullanılan Anason (Antalya/Korkuteli), Hayıt (İzmir-Torbalı), Kestane (Ordu-Fatsa), Ayçiçeği (Adana-Ceyhan), Geven (Konya-
Hadim), Çakşır (Mersin-Silifke), Korunga (Van-Başkale), Orman gülü (Giresun-Bulancak), Ihlamur (KastamonuAzdavay) ve Maydanoz balı (Hatay-İskenderun) örnekleri 2013 yılında arıcı birlikleri vasıtasıyla üreticilere ulaşılarak her bal örneği için 3 tekerrürlü olarak temin edilmiştir. Ballar serin ve güneş almayacak şekilde analizler yapılıncaya kadar $4^{\circ} \mathrm{C}^{\prime}$ de saklanmış, analizden 1 gece öncesinde oda sicaklığında $\left(24 \pm 2^{\circ} \mathrm{C}\right)$ bekletildikten sonra analize tabi tutulmuştur. (Pehlivan ve Gül, 2015). Balların botanik kökenleri, Maurizio (1951) tarafindan bildirilen polen analizi yöntemi ile tespit edilmiştir. Baldaki yoğunluğu $\% 45$ ve daha fazla olan polenler dominant polen olarak değerlendirilmiştir (Jhansi ve ark, 1991).

Monofloral bal örneklerinde yapılan biyokimyasal analizler (kül, elektriksel iletkenlik pH, nem, asitlik, HMF, diyastaz, invert şeker ve sakkaroz) Anonymous, (1995) ve Bogdanov ve ark, (1997) belirttiği yöntemlere göre yapılmıştır. Veriler SPSS paket programında (General Linear ModellUnivariate) analiz edilmiş ve her değişkene ait ortalamalar Duncan çoklu karşılaştırma testi ile karşılaştırılmıştır.

\section{Bulgular ve Tartışma}

Çalışma kapsamında ilk etapta bal örneklerinin botanik kökenlerini belirlemek amacıyla polen analizleri yapılmıştır. Yapılan mikroskobik sayımlar sonunda bal örneklerindeki dominant polen sayısı ortalamaları Anason (Pimpinella sp.) için \%45, Kestane (Castanea sp.) \%75, Geven (Astragalus sp.) balında \%52, Korunga (Onobrychis sp.) \%47, Maydanoz (Petroselinum sp.) $\% 79$, Hayıt (Vitex agnus-castus) \%86, Ayçiçeği (Helianthus annuus) \%86, Ihlamur (Tilia sp.) \%66, Orman gülü (Rhododendron sp.) \%56 ve Çakşır (Ferula sp.) balı için \%47 olarak tespit edilmiştir. Bal örneklerinde $\% 45$ ve üzeri aynı bitki polen içeriğine sahip ballar monofloral ballar olarak kabul edilmiştir. Örnekler içerisinde ayçiçeği ve hayıt balları (\%86) en yüksek oranda botanik kökenini temsil etmektedir. En düşük polen frekansı ile temsil edilen monofloral bal örneği ise $\% 45$ ile anason balıdır.

Çalışmada yer alan monofloral ballara ait biyokimyasal analiz sonuçları Tablo 1'de verilmiştir. Tablo 1'de görüldüğü üzere bal örneklerinin ortalama nem oranları \%19,15 olarak bulunmuştur. Bu değer Türk Gıda Kodeksi Bal Tebliği, Avrupa Birliği ve Kodeks standartlarında belirtilen en fazla \%20 sınırının altındadır. Nem oranı \%20'nin üzerinde bulunan kestane balının nem içeriği doğal özelliğinden kaynaklanmakta ve genellikle nem oranı yüksek bulunmaktadır (Gül, 2008). Ancak maydanoz ve ayçiçeği ballarının erken hasat nedeniyle tam olarak olgunlaşmadığı ve bu nedenle nem içeriklerinin yüksek olduğu düşünülmektedir. Zira petek yüzeyin $1 / 2$ ya da $2 / 3$ 'ü sırlanmadan hasat edilen balların nem içerikleri yüksek bulunmaktadır (Genç ve Dodoloğlu, 2011; Doğaroğlu, 1999). Yapılan istatistiki analizler sonucunda bal örneklerinde belirlenen kül, nem, ph, asitlik, elektriksel iletkenlik, HMF, Diastaz sayısı, invert şeker ve sakkaroz miktarı ortalamaları arasındaki farklar istatistiki olarak önemli bulunmuştur $(\mathrm{P}<0,000)$.

Çalışmada toplanan monofloral bal örneklerinin \% kül miktarları 0,14-0,59 arasında değişmekte olup ortalama $0,37 \pm 0,04$ olarak belirlenmiştir. Kül miktarına bağlı olarak hesaplanan elektriksel iletkenlik değerleri ise 0,38 - 
1,17 arasında değişmekte olup ortalama $0,79 \pm 0,07$ olarak hesaplanmıştır. Balların $\mathrm{pH}$ içeriğine ilişkin ulusal ya da uluslararası bir standart değer bulunmamakla beraber yapılan analizler sonucunda örneklerin $\mathrm{pH}$ değerleri ortalama 3,97 $\pm 0,02$ olarak tespit edilmiştir. Araştırmada yer alan monofloral balların asitlik miktarı ise ortalama $29,07 \pm 0,39 \mathrm{meq} / \mathrm{kg}$ olarak belirlenmiştir. Bu değer Türk Gıda Kodeksi Bal Tebliği, Kodeks ve Avrupa Birliği (EU) standartlarında çiçek ve salgı balı için belirtilen $<50$ $\mathrm{meq} / \mathrm{kg}$ sınır değeri ile uygun bulunmuştur.
Balların Hidroksimetilfurfural (HMF) içerikleri ve diyastaz sayıları da ortalama $6,17 \pm 0,76 \mathrm{mg} / \mathrm{kg}$ ve $22,05 \pm 0,00$ olarak bulunmuştur. Çalışmada yer alan örneklere ait HMF miktarının Türk Gıda Kodeksi Bal Tebliği, Avrupa Birliği standardı ve Kodeks ( $<40$ mg/kg), standartlarına uygun olduğu saptanmıştır. Ayrıca diyastaz sayısı bakımından da örneklerin FAO/G1da Kodeksi, Türk Gıda Kodeksi Bal Tebliği ve Avrupa Birliği standardının belirlediği en az limit olan 8'in üzerinde olduğu görülmektedir.

Tablo 1 Bal örneklerinin biyokimyasal analiz sonuçları(ort. \pm SE)

\begin{tabular}{|c|c|c|c|c|c|}
\hline Bal Örnekleri & $\begin{array}{l}\text { Kül } \\
(\%)\end{array}$ & $\begin{array}{c}\text { Elektriksel İletkenlik } \\
\mathrm{mS} / \mathrm{cm}\end{array}$ & $\begin{array}{c}\text { Nem } \\
(\%)\end{array}$ & $\mathrm{pH}$ & $\begin{array}{c}\text { Asitlik } \\
(\mathrm{meq} / \mathrm{kg})\end{array}$ \\
\hline Geven & $0,50^{\mathrm{ab}^{*}} \pm 0,04$ & $1,02^{\mathrm{ab}} \pm 0,08$ & $17,47^{\mathrm{e}} \pm 0,06$ & $3,95^{\mathrm{d}} \pm 0,02$ & $43,47^{\mathrm{a}} \pm 0,21$ \\
\hline Korunga & $0,41^{\mathrm{bc}} \pm 0,04$ & $0,87^{\mathrm{bc}} \pm 0,07$ & $18,47^{\mathrm{d}} \pm 0,15$ & $4,19^{c} \pm 0,03$ & $36,33^{\mathrm{c}} \pm 0,35$ \\
\hline Ihlamur & $0,32^{c} \pm 0,03$ & $0,70^{\mathrm{c}} \pm 0,06$ & $17,90^{\mathrm{d}} \pm 0,18$ & $4,56^{\mathrm{b}} \pm 0,01$ & $41,27^{\mathrm{b}} \pm 0,42$ \\
\hline Orman gülü & $0,48^{\mathrm{ab}} \pm 0,03$ & $0,98^{a b} \pm 0,05$ & $19,10^{\mathrm{c}} \pm 0,00$ & $3,23^{\mathrm{e}} \pm 0,01$ & $16,77^{\mathrm{h}} \pm 0,93$ \\
\hline Hayit & $0,17^{\mathrm{d}} \pm 0,07$ & $0,43^{\mathrm{d}} \pm 0,12$ & $18,23^{\mathrm{d}} \pm 0,08$ & $3,94^{\mathrm{d}} \pm 0,01$ & $23,53^{\mathrm{g}} \pm 0,20$ \\
\hline Çakşır & $0,35^{\mathrm{c}} \pm 0,05$ & $0,75^{\mathrm{c}} \pm 0,10$ & $17,83^{\mathrm{e}} \pm 0,21$ & $4,23^{c} \pm 0,01$ & $28,27^{\mathrm{d}} \pm 0,32$ \\
\hline Kestane & $0,59^{\mathrm{a}} \pm 0,06$ & $1,17^{\mathrm{a}} \pm 0,11$ & $20,70^{\mathrm{b}} \pm 0,10$ & $4,77^{\mathrm{a}} \pm 0,01$ & $23,40^{\mathrm{g}} \pm 0,35$ \\
\hline Anason & $0,57^{\mathrm{a}} \pm 0,03$ & $1,13^{\mathrm{a}} \pm 0,06$ & $19,00^{\mathrm{c}} \pm 0,02$ & $4,12^{c} \pm 0,03$ & $26,27^{\mathrm{f}} \pm 0,21$ \\
\hline Ayçiçeği & $0,14^{\mathrm{d}} \pm 0,02$ & $0,38^{\mathrm{d}} \pm 0,05$ & $20,23^{\mathrm{b}} \pm 0,06$ & $3,35^{\mathrm{e}} \pm 0,01$ & $27,13^{\mathrm{e}} \pm 0,35$ \\
\hline Maydanoz & $0,17^{\mathrm{d}} \pm 0,02$ & $0,43^{\mathrm{d}} \pm 0,04$ & $22,53^{\mathrm{a}} \pm 0,05$ & $3,38^{\mathrm{e}} \pm 0,02$ & $24,23^{\mathrm{g}} \pm 0,63$ \\
\hline $\mathrm{En} \mathrm{Az}$ & 0,14 & 0,38 & 17,47 & 3,23 & 16,77 \\
\hline En Çok & 0,59 & 1,17 & 22,53 & 4,77 & 43,47 \\
\hline Ortalama & $0,37 \pm 0,04$ & $0,79 \pm 0,07$ & $19,15 \pm 0,09$ & $3,97 \pm 0,02$ & $29,07 \pm 0,39$ \\
\hline $\mathrm{P}$ & 21,60 & 21,60 & 63,76 & 103,95 & 977,14 \\
\hline $\mathrm{F}$ & 0,000 & 0,000 & 0,000 & 0,000 & 0,000 \\
\hline TGKBT (Anonim,2013) & $\leq 0,6(\mathrm{CB}) \leq 1,0(\mathrm{SB})$ & $\leq 0,8(\mathrm{CBB}) \geq 0,8(\mathrm{SB})$ & $\leq 20 \%$ & & $\leq 50(\mathrm{CBB}) \leq 50(\mathrm{SB})$ \\
\hline AB (Bogdanov et all, 1997) & $<0,6(\mathrm{CB})<1,0(\mathrm{SB})$ & $<0,8(\mathrm{CB})>0,8(\mathrm{SB})$ & $<20 \%$ & & $<50(\mathrm{CB})<50(\mathrm{SB})$ \\
\hline Kodeks (Anony., 1995) & $\leq 0,6(\mathrm{ÇB}) \leq 1,0(\mathrm{SB})$ & $\leq 0,8(\mathrm{C} B)>0,8(\mathrm{SB})$ & $\leq 20 \%$ & & $\leq 50(\mathrm{CB}) \leq 50(\mathrm{SB})$ \\
\hline \multicolumn{6}{|c|}{ *: Aynı sütundaki farklı harfler ortalamalar arasında önemli fark olduğunu göstermektedir (Duncan, $\mathrm{P}<0,000) \mathrm{SB}=\mathrm{Salg}$ 1 Balı; ÇB : Çiçek Balı } \\
\hline Bal Örnekleri & HMF $(\mathrm{mg} / \mathrm{kg})$ & Diyastaz Say1s1 & \multicolumn{2}{|c|}{ İnvert Şeker $(\%)$} & Sakkaroz (\%) \\
\hline Geven & $12,29^{\mathrm{a}} \pm 1,45$ & $17,9 \pm 0,00$ & \multicolumn{2}{|c|}{$70,56^{\mathrm{b}} \pm 0,17$} & $3,31^{\mathrm{a}} \pm 0,24$ \\
\hline Korunga & $8,64^{\mathrm{b}} \pm 1,07$ & $29,4 \pm 0,00$ & \multicolumn{2}{|c|}{$70,89^{\mathrm{bc}} \pm 0,22$} & $3,27^{\mathrm{a}} \pm 0,33$ \\
\hline Ihlamur & $5,06^{c} \pm 1,22$ & $17,9 \pm 0,00$ & \multicolumn{2}{|c|}{$74,08^{\mathrm{a}} \pm 0,74$} & $2,67^{b} \pm 0,54$ \\
\hline Orman gülü & $3,92^{\mathrm{c}} \pm 0,75$ & $17,9 \pm 0,00$ & \multicolumn{2}{|c|}{$67,73^{\mathrm{e}} \pm 0,38$} & $2,32^{\mathrm{b}} \pm 0,09$ \\
\hline Hayit & $12,62^{\mathrm{a}} \pm 0,95$ & $17,9 \pm 0,00$ & \multicolumn{2}{|c|}{$69,01^{\mathrm{d}} \pm 0,45$} & $3,14^{\mathrm{a}} \pm 0,80$ \\
\hline Çakşır & $2,39^{\mathrm{d}} \pm 0,38$ & $29,4 \pm 0,00$ & \multicolumn{2}{|c|}{$70,83^{\mathrm{c}} \pm 0,91$} & $2,27^{\mathrm{b}} \pm 0,21$ \\
\hline Kestane & $4,61^{\mathrm{c}} \pm 0,69$ & $29,4 \pm 0,00$ & \multicolumn{2}{|c|}{$69,77^{\mathrm{d}} \pm 0,56$} & $1,89^{\mathrm{b}} \pm 0,28$ \\
\hline Anason & $4,52^{\mathrm{c}} \pm 0,40$ & $17,9 \pm 0,00$ & \multicolumn{2}{|c|}{$69,57^{\mathrm{d}} \pm 0,49$} & $3,58^{\mathrm{a}} \pm 0,37$ \\
\hline Ayçiçeği & $4,10^{c} \pm 0,29$ & $17,9 \pm 0,00$ & \multicolumn{2}{|c|}{$67,46^{\mathrm{e}} \pm 0,42$} & $3,20^{\mathrm{a}} \pm 0,11$ \\
\hline Maydanoz & $3,50^{\mathrm{cd}} \pm 0,37$ & $29,4 \pm 0,00$ & \multicolumn{2}{|c|}{$67,28^{\mathrm{e}} \pm 0,61$} & $3,35^{a} \pm$ \\
\hline En $\mathrm{Az}$ & 2,39 & 17,9 & \multicolumn{2}{|c|}{67,28} & 1,89 \\
\hline En Çok & 12,62 & 29,4 & \multicolumn{2}{|c|}{74,08} & 3,58 \\
\hline Ortalama & $6,17 \pm 0,76$ & $22,05 \pm 0,00$ & \multicolumn{2}{|c|}{$69,72 \pm 0,49$} & $2,90 \pm 0,30$ \\
\hline $\mathrm{P}$ & 56,56 & - & \multicolumn{2}{|c|}{315,37} & 8,31 \\
\hline $\mathrm{F}$ & 0,000 & - & \multicolumn{2}{|c|}{0,000} & 0,000 \\
\hline TGKBT (Anonim,2013) & $\leq 40(\mathrm{CB}) \leq 40(\mathrm{SB})$ & $\geq 8(\mathrm{CB}) \geq 8(\mathrm{SB})$ & \multicolumn{2}{|c|}{$\geq 60(\mathrm{CB}) \geq 45(\mathrm{SB})$} & $\leq 5(\mathrm{CBB}) \leq 10(\mathrm{SB})$ \\
\hline AB (Bogdanov et all, 1997) & $\leq 40(\mathrm{CB}) \leq 40(\mathrm{SB})$ & $\geq 8(\mathrm{CB}) \geq 8(\mathrm{SB})$ & \multicolumn{2}{|c|}{$\geq 60(\mathrm{CB}) \geq 45(\mathrm{SB})$} & $\leq 5(\mathrm{CB}) \leq 10(\mathrm{SB})$ \\
\hline Kodeks (Anony., 1995) & $\leq 40(\mathrm{C} B) \leq 40(\mathrm{SB})$ & $\geq 8(\mathrm{C} B) \geq 8(\mathrm{SB})$ & \multicolumn{2}{|c|}{$>60(\mathrm{ÇB})>45(\mathrm{SB})$} & $\leq 5(\mathrm{ÇB}) \leq 10(\mathrm{SB})$ \\
\hline
\end{tabular}

*: Aynı sütundaki farklı harfler ortalamalar arasında önemli fark olduğunu göstermektedir (Duncan, $\mathrm{P}<0,000$ ) $\mathrm{SB}=$ Salgı Balı; ÇB : Çiçek Balı

Tablo 2 Balda yapılan bazı biyokimyasal analiz çalışmaları

\begin{tabular}{l|cccccccc}
\hline \multicolumn{1}{c}{ Parametreler } & Sonuç** & KAY1 & KAY2 & KAY3 & KAY4 & KAY5 & KAY6 & KAY7 \\
\hline Kül (\%) & $0,37 \pm 0,04$ & $0,1-1,59^{*}$ & $0,01-0,12$ & - & $0,04-1,72^{*}$ & $0,30^{*}$ & 0,06 & $0,32^{*}$ \\
E.iletkenlik & $0,79 \pm 0,07$ & $0,31-2,49^{*}$ & & $0,2-0,9^{*}$ & $0,22-3,14^{*}$ & $0,67^{*}$ & - & $0,69^{*}$ \\
Nem (\%) & $19,15 \pm 0,09$ & - & $17,1-20,0^{*}$ & $16-17,25$ & $12,12-27,4$ & $19,11^{*}$ & $18,4^{*}$ & 16,03 \\
pH & $3,97 \pm 0,02$ & $3,49-4,91^{*}$ & - & - & $3,03-4,73^{*}$ & $3,59^{*}$ & - & $4,12^{*}$ \\
Asitlik (meq/kg) & $29,07 \pm 0,39$ & $39,28-32,46^{*}$ & $18,2-47,5^{*}$ & - & $18,0-145,5^{*}$ & $28,19^{*}$ & - & 40,41 \\
Diyastaz & $22,05 \pm 0,00$ & - & $10,9-17,9$ & - & - & $22,81^{*}$ & - & 10,31 \\
HMF (mg/kg) & $6,17 \pm 0,76$ & - & $1,34 *-31,28$ & - & $2,21^{*}-229,6^{*}$ & $8,44^{*}$ & 14,8 & - \\
İnvert şeker (\%) & $69,72 \pm 0,49$ & - & $51,31-68,30^{*}$ & - & - & $71,67^{*}$ & - & 57,83 \\
Sakkaroz(\%) & $2,90 \pm 0,30$ & - & - & - & - & $2,24^{*}$ & - & 2,39 \\
\hline
\end{tabular}

*: Çalışmada elde edilen veriler ile uyumlu ya da yakın değerleri temsil etmektedir, **Sonuç: ort.土SE, KAY1: Karabagias ve ark. (2014), KAY2: Özcan ve Ölmez (2013), KAY3: Kowalski (2013), KAY4: Alqarni ve ark. (2012), KAY5: Gül, (2008), KAY6: Finola ve ark. (2007), KAY7: Şahinler ve ark. (2004) 
Çalışmada, balların içerdikleri invert şeker (glikoz + früktoz) ve sakkaroz miktarı da Tablo 1'de verilmiştir. Bal örneklerinde yapılan şeker analizi sonucunda invert şeker miktarının ortalama \%69,73 olduğu tespit edilmiştir. Elde edilen invert şeker içeriğinin Türk Gıda Kodeksi Bal Tebliği, Kodeks ve Avrupa Birliği (>60 (ÇB) >45 (SB) standartlarına uygun olduğu belirlenmiştir. Ballardaki sakkaroz miktarı ise ortalama \%2,68 olarak belirlenmiștir. Analiz sonucunda elde edilen sakkaroz değerleri Türk Gıda Kodeksi Bal Tebliği, Avrupa Birliği Standardı ve Kodeks standartlarında belirtilen çiçek ballarında en çok $\% 5$, çam ballarında ise en çok \%10 sınırına uygun bulunmuştur. Çalışmada elde edilen verilerin diğer yapılan bazı çalışmalar ile karşılaştırmaları Tablo 2'de yapılmıştır. Sonuçlar incelendiğinde, bu çalışmada yer alan bal örneklerinin biyokimyasal özellikler bakımından genel olarak ulusal ve uluslararası standartlara uygun olduğu ve tabloda verilen diğer çalışmalara benzerlik gösterdiği tespit edilmiştir.

Sonuç olarak Türkiye'de üretilen bazı monofloral balların biyokimyasal içeriklerinin Türk Gida Kodeksi Bal Tebliği (Anonim, 2013), Avrupa Birliği Standardı (Bogdanov ve ark., 1997) ve Kodeks Standartlarına (Anonim, 1995) uygun olduğu tespit edilmiştir. Genel anlamda tüm bal örneklerinde yapılan analizler sonucunda elde edilen değerler birbirine yakın, ancak istatistiki olarak birbirinden farklı bulunmuştur. (Tablo 2). Ancak bal örneklerinde antioksidan ve antimikrobiyal analizler gibi daha spesifik analizlerin yapılması ile daha çarpıcı sonuçlar elde edilebilir. Biyokimyasal özellikler bakımından uygun nitelikteki balların tüketiciye sunulması halk sağlığ 1 ve bu önemli gıda maddesinden insanların etkin biçimde faydalanabilmesi bakımından önem taşımaktadır.

\section{Kaynaklar}

Aljadi AM, Kamaruddin MY. 2004. Evaluation of the Phenolic Contents and Antioxidant Capacities of two Malaysian Floral Honeys. Food Chem., 85 (4): 513-518.

Alqarni AS, Owayss AA, Mahmoud AA, Hannan MA. 2014. Mineral Content and Physical Properties of Local and Imported Honeys in Saudi Arabia. J. of Saudi Chem. Soc., 18(5): 618-625.

Anonim. 1995. Official Methods of Analysis of AOAC International. 16th Ed. (Patricia A.C., Editor), ed. P. Cunniff. AOAC International, Arlington, Virginia, USA.

Anonim. 2013. Türk Gıda Kodeksi Bal Tebliği. Tebliğ No: 201/582. http://www.resmigazete.gov.tr/eskiler/2012/07/ 20120727-12.htm. Erişim tarihi: 14.10.2016.

Bogdanov S, Martin P, Lullmann C. 1997. Harmonized Methods of the European Honey Commission. Apidologie, extra issue. 1-59.

Bogdanov S, Jurendic T, Sieber R. 2008. Honey for Nutrition and Health: A Review. Am J Coll Nutr, 27(6): 677-689.

Cavia MM, Fernandez-Muino MA, Gömez-Alonso E, MontesPerez MJ, Huidobro JF and Sancho MT. 2002. Evolution of Fructose and Glucose in Honey over one year: Influence of Induced Granulation. Food Chem., 78(2):157-161.

Chua LS, Adnan NA. 2014. Biochemical and Nutritional Components of Selected Honey Samples. Acta Sci. Pol., Technol. Aliment, 13(2):169-179.

Doğaroğlu M. 1999. Modern Arıcılık Teknikleri. Anadolu Matbaa, Tekirdağ, 1999.

Furatlı Ç, Genç F, Karacaoğlu M, Gençer HV. 2000. Türkiye Arıcılığının Karşılaştırmalı Analizi, Sorunlar, Öneriler. Türkiye Ziraat Mühendisliği V. Teknik Kongresi Bildirileri, Ankara.
Finola MS, Lasagno MC, Marioli JM. 2007. Microbiological and chemical characterization of honeys from central Argentina. Food Chem., 100(4): 1649-1653

Genç F, Dodoloğlu A. 2011. Arıcılığın Temel Esasları. Atatürk Üniversitesi, Ziraat Fakültesi, Zootekni Bölümü, Yayın No:931-341-88, Erzurum, 386s.

Gül A. 2008. Türkiye'de Üretilen Bazı Balların Yapısal Özelliklerinin Gıda Güvenliği Bakımından Araştırılması. Mustafa Kemal Üniversitesi Fen Bilimleri Enstitüsü yayınları. Doktora Tezi, Hatay.

Jhansi P, Kaplana TP, Ramanujam CGK. 1991. Pollen Analyis of Rock Bee Summer Honeys From The Prakasam District ff The Andhra Pradesh, India. J.of Apic. Res., 30 (1): 33-40.

Kaftanoğlu O, Özgür F. 2000. Ziraai Mücadele İlaçlarının Koloniler ve Arı Ürünleri Üzerine Etkileri. Türkiye 3. Arıc1lık Kongresi. S:23. Adana

Kalpaklığlu N. 2000. Bal Üretiminde Karşılaşılan Sorunlar ve Ülke İhracatına Etkisi. Türkiye III. Arıcılık Kongresi, Bildiri Özetleri, 1-3 Kasım 2000. Adana.

Karabagias IK, Badeka AV, Kontakos S, Karabournioti S, Kontominas MG. 2014. Botanical Discrimination of Greek Unifloral Honeys with Physico-Chemical and Chemometric Analyses. Food Chem., 165: 181-190.

Karadal F, Yıldırım Y. 2012. Balın Kalite Nitelikleri, Beslenme ve Sağlık Açısından Önemi. Erciyes Üniv. Vet. Fak. Derg., 9: 197-209.

Kaskoniene V, Venskutonis PR, Ceksteryte V. 2010 Carbohydrate Composition and Electrical Conductivity of Different Origin Honeys from Lithuania. LWT-Food Sci. Technol. 43(5): 801-807.

Kowalski S. 2013. Changes of Antioxidant Activity and Formation of 5-Hydroxymethylfurfural in Honey During Thermal and Microwave Processing. Food Chem., 141(2): 1378-1382,

Maurizio A. 1951. Pollen Analysis of Honey. Bee World, 32(1): 1-5.

Nasuti C, Gabbianelli R, Falcioni G, Cantalamess F. 2006. Antioxidative and Gastroprotective Activities of AntiInflammatory Formulations Derived from Chestnut Honey in Rats. Nutr. Res., 26(3): 130-137.

Özcan MM, Ölmez Ç. 2013. Some Qualitative Properties of Different Monofloral Honeys. Food Chem., 163: 212-218.

Pehlivan T, Gül A. 2015. Determination of Heavy Metals Contents of Some Monofloral Honey Produced in Turkey. J. of App. Pharm. Sci. Vol. 5 (08): 042-045,

Silici S, Sagdic O, Ekici, L. 2010.Total Phenolic Content, Antiradical, Antioxidant and Antimicrobial Activities of Rhododendron Honeys. Food Chem., 121(1): 238-243.

Şahinler N, Şahinler S, Gül A. 2004. Biochemical Composition of Honeys Produced in Turkey. J. of Apic. Res., 43(2): 53-56.

Vela L, De Lorenzo C, Pérez RA. 2007. Antioxidant Capacity of Spanish Honeys and its Correlation with the Polyphenolic Content and Other Physico-Chemical Properties. J of the Sci. of Food and Agri., 87(6): 1069-1075.

Wang H. 2011. The Study of the Antioxidant Activity of Phenolic Components of Manuka Honey. The University of Waikato. Master of Science in Biological Sci. Hamilton, New Zealand.

Weston RJ. 2000. The Contribution of Catalase and Other Natural Products to the Antibacterial Activity of Honey: A review. Food Chem., 71(2): 235-239.

White JW JR, Riethof ML, Kushnir L. 1961. Composition of Honey. VI. The Effect of Storage on Carbohydrates, Acidy and Diastase Content. J. of Food Sci. 26(1): 63-66.

White JW, Subers MH, Shepart AY.1963. The Identification of Inhibine, the Antibacterial Factor in Honey, as Hydrogen Peroxide, and its Origin in a Glucose-Oxidase System. Biochim. Biophys. Acta, 73(1): 57-70. 